Rev. Elev. Méd. vét. Pays trop., 1968, 21, 4 (463-466).

\title{
Note sur la résistance au Bérénil d'une souche tchadienne de Trypanosoma vivax
}

\author{
por M. GRABER
}

\begin{abstract}
RÉSUMÉ
L'auteur ayant pris connassance d'une observation récente faite à Kaduna (Nigeria du nord) rappelle qu'en 1956 avaił déjà été sıgnalé au Tchad un cas semblable de résistance au bérénıl d'une souche de Trypanosoma vivax.
\end{abstract}

Le problème de la résistance au Bérénil de certaines souches de Trypanosoma vivax est d'autant plus important que plusieurs Etats d'Afrique centrale emploient de plus en plus ce trypanocide dans la lutte contre la Trypanosomiase animale (Tchad, Cameroun).

On sait que le médicament, s'il est sans action sur $T$. simice, $T$. evansı ou même $T$, brucei, est capable de détruire $T$. congolense et, à un degré moindre T. vivax (FINELLE 1957 ; FAIRCLOUGH 1960 ef 1962).

Tout récemment, dans une lettre au Rédacteur en chef du Veterinary Record, JONES DAVIES (1967) signale chez des zébus Foulani du centre d'essai de Kaduna (Nigeria du Nord), l'existence d'un Trypanosoma vivox résistant au Bérénil.

Or, dès 1956, le même phénomène avait été observé ef décri† au laboratoire de Farcha (Rapport annuel).

Pour éviter des erreurs de traitement dans des régions où $T$. vivox ef $T$. brucei sont les espèces dominantes, comme le Sud-Ouest du Tchad (TRONCY 1967), il a paru intéressant de rappeler les résultats obtenus au cours de cette expérience.

\section{MATÉRIEL ET MÉTHODE}

Les animaux venus de la région de Massakory en novembre 1956 éfaient fous atternts d'une infection à Tryponosoma vivax plus ou moins forte.
Ils ont été placés, dès leur arrivée, dans des étables cimentées et soustraits, autant que faire se peut à l'action des insectes piqueurs, par des lotions répétées d'lxogal. L'essai a duré 1 mois et demı, du 15 novembre 1956 av début janvier 57.

41 bouvillons de 10 mois à 30 mois ont été ainsi utilisés dont 9 témoins.

Le Bérénil a été administré à des doses progressivement croissantes (de 1 à $10 \mathrm{mg} / \mathrm{kg}$ ) par la voie sous-cutanée, par la voie intramusculaire et par la voie intraveineuse.

Dès la fin du traitement, chaque animal a été rigoureusement contrôlé. Des lames de sang colorées au Giemsa (goutte épaisse) ont été faites une fois par jour.

Les animaux traltés ef morts, sans que les Trypanosomes aient réapparu, ont subi des examens nécropsiques minutieux, en particulier des ganglions et de certains organes (foie ef rate).

\section{RÉSULTATS}

Ils sont groupés dans le tableau no 1 . Quelle que soit la voie d'introduction du médicament, Trypanosoma vivax, après traitement au Bérénil, réapparaît :

- entre le $8^{\mathrm{e}}$ ef le $14^{\mathrm{e}}$ jour à $1 \mathrm{mg} / \mathrm{kg}$.

- entre le $9 e$ et le $19 \mathrm{e}$ jour à $2 \mathrm{mg} / \mathrm{kg}$,

- entre le $12^{e}$ et le $18^{e}$ jour à $3 \mathrm{mg} / \mathrm{kg}$,

- vers le 18 e jour à $4 \mathrm{mg} / \mathrm{kg}$,

- entre le $14^{\mathrm{e}}$ et le $20 \mathrm{e}$ jour à $5 \mathrm{mg} / \mathrm{kg}$. 
TABLEAU $\mathrm{N}^{0}$ I

a) Animaux têmolns

\begin{tabular}{|c|l|c|}
\hline $\begin{array}{c}\text { Bouvillon } \\
\mathrm{N}^{\mathrm{a}}\end{array}$ & Infestation & Mort \\
\hline 19 & Forte & 3 ème jour \\
20 & Très massive & 3 ème jour \\
21 & Moyenne & 9 ème jour \\
22 & Faible & 23 ème jour \\
23 & Faible & 29 ème jour \\
24 & Falble & 7 ème jour \\
25 & Très massive & 2 ème jour \\
26 & Moyenne & 6 ème jour \\
27 & Moyenne & 7 ème jour \\
\hline
\end{tabular}

- A $7 \mathrm{mg} / \mathrm{kg}$, sur 3 animaux, l'un se montre positif le $32 \mathrm{e}$ jour, alors que les deux autres bouvillons examinés le $20 \mathrm{e}$ et le $43 \mathrm{e}$ jour demeurent négatifs.

Les observations de JONES DAVIES (1967) portent sur 11 animaux artificiellement infestés par Trypanosoma vivax et entretenus dans une étable grillagée, à l'abri des mouches, de septembre 1966 d̀ janvier 1967. L'auteur constate qu'à la dose classique de $3,5 \mathrm{mg} / \mathrm{kg}$, il y a rechute 14 et 20 jours après le traitement. A $7 \mathrm{mg} / \mathrm{kg}$, il est possible de retrouver Trypanosoma vivax 14 et 16 jours plus tard sur les animaux maintenus en étable et, au bout de deux mois dans l'ensemble du troupeau de la station de Kaduna.

TABLEAU N ${ }^{\circ} \mathrm{I}$ (suite)

Traitement au Bêrëril

b) Animaux traités

\begin{tabular}{|c|c|c|c|c|}
\hline $\begin{array}{c}\text { Bouvilion } \\
\mathrm{N}^{\circ}\end{array}$ & $\begin{array}{l}\text { Doses } \\
\mathrm{mg} / \mathrm{kg}\end{array}$ & $\begin{array}{l}\text { Infestation avant } \\
\text { traitement }\end{array}$ & $\begin{array}{c}\text { Réapparftion des Trypanosomes } \\
\text { après traítement }\end{array}$ & Mortalité \\
\hline $\begin{array}{l}\text { Vole intrave } \\
\qquad \begin{array}{c}2 \\
3 \\
14 \\
16 \\
37 \\
41\end{array}\end{array}$ & $\begin{array}{r}1 \\
2 \\
4 \\
5 \\
7 \\
10\end{array}$ & $\begin{array}{c}\text { Faible } \\
" \\
" \\
" \\
" \\
\text { Moyenne }\end{array}$ & $\begin{array}{l}8 \text { ème jour } \\
19 \text { ème jour } \\
18 \text { ère jour } \\
20 \text { ème four } \\
32 \text { ème jour } \\
\text { aucune le } 12 \text { ème jour }\end{array}$ & Mort \\
\hline $\begin{array}{c}\text { Voie intramu } \\
\qquad \begin{array}{r}1 \\
29 \\
6 \\
30 \\
9 \\
13 \\
34 \\
15 \\
38 \\
39\end{array}\end{array}$ & $\begin{array}{r}1 \\
1 \\
2 \\
3 \\
3 \\
4 \\
4 \\
5 \\
7 \\
10\end{array}$ & $\begin{array}{l}\text { Faible } \\
\text { Masslve } \\
\text { Masslve } \\
\text { Moyenne } \\
\text { Forte } \\
\text { Moyenne } \\
\text { Faible } \\
\text { Moyenne } \\
\text { Moyenne } \\
\text { Faible }\end{array}$ & $\begin{array}{l}14 \text { ème jour } \\
\text { aucune le } 10 \text { ème jour } \\
16 \text { ème four } \\
\text { aucune le } 5 \text { ème jour } \\
12 \text { ềme jour } \\
1 B \text { ème jour } \\
\text { aucune le } 4 \text { ème jour } \\
22 \text { ème jour } \\
\text { aucune le } 43 \text { ème jour } \\
\text { aucune le } 8 \text { ème jour }\end{array}$ & $\begin{array}{l}\text { Mort } \\
\text { Mort } \\
\text { Mort } \\
\text { Mort } \\
\text { Mort }\end{array}$ \\
\hline $\begin{array}{l}\text { Voie sous-cu } \\
28 \\
4 \\
5 \\
7 \\
8 \\
11 \\
10 \\
12 \\
31 \\
32 \\
33 \\
35 \\
17 \\
18 \\
36 \\
40\end{array}$ & $\begin{array}{r}1 \\
2 \\
2 \\
2 \\
3 \\
3 \\
3 \\
3 \\
3 \\
3 \\
4 \\
5 \\
5 \\
5 \\
7 \\
10\end{array}$ & $\begin{array}{l}\text { Moyenne } \\
\text { Faible } \\
\text { Massive } \\
\text { Massive } \\
\text { Moyenne } \\
\text { Forte } \\
\text { Moyenne } \\
\text { Moyenne } \\
\text { Moyenne } \\
\text { Forte } \\
\text { Moyenne } \\
\text { Faible } \\
\text { Faible } \\
\text { Moyenne } \\
\text { Faible } \\
\text { Faible }\end{array}$ & $\begin{array}{l}\text { aucune le } 10 \text { ème jour } \\
9 \text { ème jour } \\
13 \text { ème jour } \\
14 \text { ème jour } \\
17 \text { ème jour } \\
17 \text { ème jour } \\
15 \text { ème jour } \\
18 \text { ème jour } \\
\text { aucune le } 5 \text { ème jour } \\
\text { aucume le } 14 \text { ême joưr } \\
\text { aucune le } 4 \text { ème jour } \\
\text { aucune le } 3 \text { ème jour } \\
19 \text { ème jour } \\
14 \text { ème jour } \\
\text { aucune le } 20 \text { ème jour } \\
\text { auccune le } 15 \text { ème jour }\end{array}$ & $\begin{array}{l}\text { Mort } \\
11 \\
11 \\
1 \\
1\end{array}$ \\
\hline
\end{tabular}

Très massive $=$ plus de 20 Trypanosomes par champ ; Massive $=$ de 10 à 20 Trypanosomes par champ; Forte $=$ de 5 à 10 Trypanosomes par champ; Falble = moins de 1 Trypanosome par champ ; 
Les essals de JONES DAVIES effectués dans des conditions plus sévères ef plus sûres qu'à Farcha (étables grillagées entre autres), mais à la même époque (fin de la saison des pluies début de la saison sèche, c'est-à-dire de septembre d̀ décembre) aboutissent sensiblement aux mêmes résultats, à savoir qu'à $3,5 \mathrm{mg} / \mathrm{kg}$, le Bérénil ne paraît détruire que très imparfaitement Trypanosoma vivax qui redevient visible dans le sang au bout de $14-20$ jours (12-18 jours à Farcha).

En doublant la dose, les résultats sont meilleurs $(7 \mathrm{mg} / \mathrm{kg}$ ). La plupart des animaux sem- blent clinquement guéris, mais sur un petit nombre d'entre eux on observe de nouveau des parasites dans un laps de temps qui va d'un mois (Farcha) à 60 jours (Kaduna).

Les Trypanosomes rencontrés après le traitement au Bérénil se présentent sous l'aspect de formes courtes, trapues, non flagellées pouvant en imposer pour une autre espèce que Tryponosoma vivax. Pour lever le doute, huit chèvres adultes, indemnes de Trypanosomiase, ont été inoculées avec le sang des bouvillons litigieux $(10 \mathrm{ml})$. Six d'entre elles ont réagi pasitivement (tableau no 2).

\section{TABLEAU $\mathbb{N}^{*} \mathrm{II}$}

Inoculation de T, vivax à des chèvres

\begin{tabular}{|c|c|c|c|}
\hline $\begin{array}{c}\text { Chèvre } \\
\mathrm{N}^{\circ}\end{array}$ & $\begin{array}{c}\text { Apparition de T. vivax ap̣ès } \\
\text { Inoculation du sang de bouvillor }\end{array}$ & $\begin{array}{c}\text { Crise } \\
\text { parasitaire }\end{array}$ & M o $\mathrm{t}$ \\
\hline 1 & 3 ème jour & 7 ème jour & 7 ème jour \\
2 & 4 ème jour & 6 ème jour & 8 ème jour \\
3 & 8 ème jour & 9 ème jour & 9 ème jour \\
4 & 6 ème jour & 8 ème jour & 9 ème jour \\
5 & 3 ème jour & 6 ème jour & 6 ème jour \\
6 & 2 ème jour & 3 ème jour & 6 ème jour \\
\hline
\end{tabular}

A l'examen, il s'est avéré qu'il s'agissait bien de Trypanosoma vivax, ce qui a été confirmé à l'époque par Messieurs les Professeurs GUILHON et EUZEBY (*).

\section{CONCLUSIONS}

Des essais entrepris à l'occasion d'une impor-

${ }^{*}$ ) Que nous tenons vivernent à remercier. tante infection à Trypanosoma vivax constatée sur des animaux d'expériences du Laboratoire ont montré qu'au Tchad la résistance au bérénil de certaines souches de Tryponosoma vivax pourrait représenter un obstacle à l'utilisation de ce médicament et qu'il éta!t donc indispensable avant tout traitement de déterminer l'espèce en cause.

Les observations faites en 1956 à Fort-Lamy, correspondent à ce qui a été constaté au Nigeria en 1967 à Kaduna.

\section{SUMMARY}

Note on the resistance to berenil of a Chad strain of Tryponosoma vivax

The author notes a recenl abservation carried out in Kaduna (Northern Nigeria) and recalls that a sımılar case of resıstance to berenil of a Tryponosoma vivax strain had been reported in 1956 in Fort-Lamy.

\section{RESUMEN}

Nota sobre la resistencia al berenil de una cepa de Triponosoma vivax del Chad

El autor nota una observación reciente hecha en Kaduna (Nigeria del norte) y recuerda que en 1956 ya se habia señalado en Chad un caso semejante de resistencia al berenil de una cepa de Triponosoma vivax. 


\section{BIBLIOGRAPHIE}

1. FAIRCLOUGH (R.). - A note on the use of Berenil at Athi-Tiva-Kenya. C. Sci. Int. Rech. Trypono, 1960, $62:$ 125-27.

2. FAIRCLOUGH (R.). - A sumary of the use of Berenil in Kenya. C. Sci. Int. Rech. Trypano, CCTA, 1962, $88: 81-6$.

3. FINELLE (P.). - Les Trypanosomes bovines dans l'Ouest de l'Oubanghui-Chari. Essais de traitement par le Bérénil. Revue Elev. Méd. vét. Pays trop., 1957, X (3) : 231-47.

4. FINELLE ( $\left.P_{1}\right)$, - Rapport sur les progrès récents de la chimiothérapie des Trypanoso- miases animales. C. Sci. Int. Rech. Trypano, CCTA, 1962, 88 : 55-67.

5. JONES-DAVIES (W. J.). - The discovery of Berenil resistant Trypanosomo vivax in Northern Nigeria. Vet. Rec., 1967, 80 (17) : 531-2.

6. Rapport annuel. Laboratoire de Farcha, 1956, PP. 59-62.

7. TRONCY (P.). - Enquête préliminaire sur la Trypanosomiase des bœufs de fraction animale du Sud-Ouest du Tchad. Rapport Laboratoire du Farcha, 1967, $50 \mathrm{p}$. 